\title{
The Orbital Period Distribution of Cataclysmic Variables Found by the SDSS
}

\author{
John Southworth ${ }^{1}$, Boris T. Gänsicke ${ }^{2} \&$ Elmé Breedt ${ }^{2}$ \\ ${ }^{1}$ Astrophysics Group, Keele University, Staffordshire, ST5 5BG, UK \\ ${ }^{2}$ Department of Physics, University of Warwick, Coventry, CV4 7AL, UK
}

\begin{abstract}
The orbital period is one of the most accessible observables of a cataclysmic variable. It has been a concern for many years that the orbital period distribution of the known systems does not match that predicted by evolutionary theory. The sample of objects discovered by the Sloan Digital Sky Survey has changed this: it shows the long-expected predominance of shortperiod objects termed the 'period spike'. The minimum period remains in conflict with theory, suggesting that the angular momentum loss mechanisms are stronger than predicted.
\end{abstract}

Keywords. stars: dwarf novae - stars: novae, cataclysmic variables - stars: white dwarfs

\section{The period spike: unveiled}

The evolution of all binary systems containing a compact object is driven by the loss of angular momentum from the orbit. Unfortunately, two of the most important mechanisms, common envelope evolution and magnetic braking, are poorly understood. A major advance in our understanding of binary evolution requires the characterisation of a large and homogeneously selected sample of close binaries, such as the population of cataclysmic variables (CVs) spectroscopically discovered by the SDSS (Szkody et al., 2002, and later papers). We are therefore undertaking a project to measure the orbital periods of all SDSS CVs. Of the 291 known systems, 153 now have reliable orbital period measurements and 46 have approximate measurements (Gänsicke et al., 2009; Dillon et al., 2008; Southworth et al., 2006, 2007ab, 2008ab, 2009, 2010ab). Fig. 1 compares the orbital period distributions of SDSS and non-SDSS CVs.

CVs evolve towards shorter orbital periods, reach a minimum value, and then bounce back to longer periods due to structural changes in the low-mass secondary star. A longstanding prediction of $\mathrm{CV}$ evolution theory is an accumulation of systems at a minimum period somewhere between 60 and $70 \mathrm{~min}$ (Rappaport, Joss \& Webbink 1982) where the evolutionary timescale becomes long and the period derivative passes through zero. Unfortunately, the observed population has persistently shown both a longer minimum period of around $80 \mathrm{~min}$ and no significant increase in the number of CVs at this period. The population of SDSS CVs, however, shows for the first time a significant excess of objects at a minimum period interval of 80-86 min (Gänsicke et al., 2009). This period spike is composed primarily of CVs which are faint and therefore have been missed by previous surveys. The position of the spike remains at a longer period than predicted, implying that the angular momentum loss is faster than expected.

On recent observing runs with the ESO New Technology Telescope, we have discovered eclipses in four faint CVs within our project. SDSS J075653.11+085831.8 shows 2 mag deep eclipses on a period of $197 \mathrm{~min}$. The system SDSS J093537.46+161950.8 has 1 mag deep eclipses on a period of $92 \mathrm{~min}$, SDSS J105754.25+275947.5 has short and deep eclipses and an orbital period of $90 \mathrm{~min}$, and SDSS J143209.78+191403.5 shows $1.5 \mathrm{mag}$ deep eclipses spaced by $169 \mathrm{~min}$ (paper in preparation). Eclipsing CVs are hugely valuable 


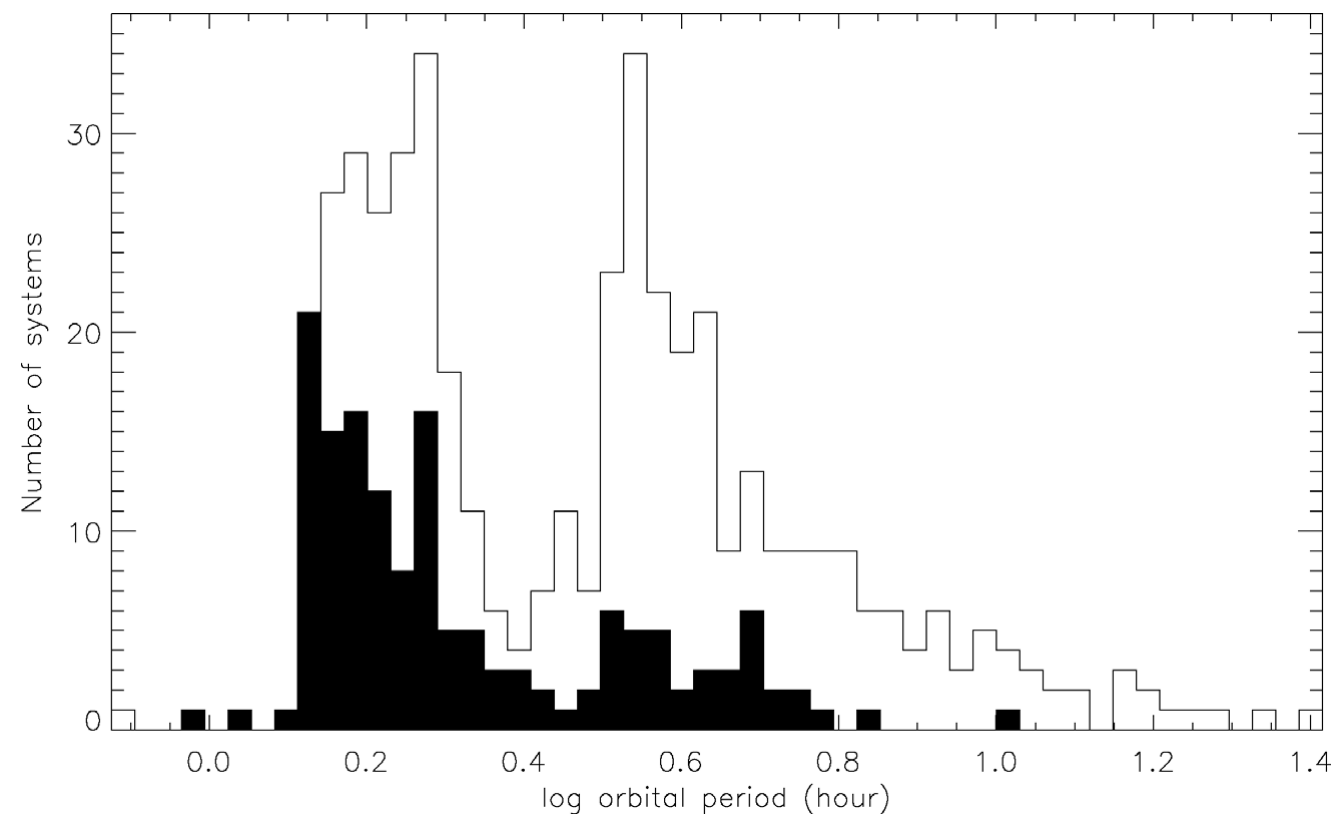

Figure 1. The orbital period distribution of SDSS CVs (black histogram) compared to that of the known non-SDSS CVs (white histogram) as catalogued by Ritter \& Kolb (2003).

because they are the only examples whose physical properties can be measured to high precision (e.g. Littlefair et al., 2006, 2008; Southworth \& Copperwheat 2011). Follow-up observations of these objects are planned.

\section{References}

Dillon, M., et al., 2008, MNRAS, 386, 1568

Gänsicke, B. T., et al., 2009, MNRAS, 397, 2170

Littlefair, S. P., Dhillon, V. S., Marsh, T. R., Gänsicke, B. T., Southworth, J., \& Watson, C. A. 2006, Science, 314, 1578

Littlefair, S. P., Dhillon, V. S., Marsh, T. R., Gänsicke, B. T., Southworth, J., Baraffe, I., Watson, C. A., \& Copperwheat, C. 2008, MNRAS, 388, 1582

Rappaport, S., Joss, P. C., \& Webbink, R. F. 1982, ApJ, 254, 616

Ritter, H. \& Kolb, U. 2003, A\&A, 404, 301

Southworth, J. \& Copperwheat, C. M. 2011, The Observatory, 131, 66

Southworth, J., Gänsicke, B. T., Marsh, T. R., de Martino, D., Hakala, P., Littlefair, S., Rodriguez-Gil, P., \& Szkody, P. 2006, MNRAS, 373, 687

Southworth, J., Gänsicke, B. T., Marsh, T. R., de Martino, D., \& Aungwerojwit, A. 2007a, $M N R A S, 378,635$

Southworth, J., Marsh, T. R., Gänsicke, B. T., Aungwerojwit, A., de Martino, D., \& Hakala, P. 2007b, MNRAS, 382, 1145

Southworth, J., et al., 2008a, MNRAS, 391, 591

Southworth, J., Townsley, D. M., \& Gänsicke, B. T. 2008b, MNRAS, 388, 709

Southworth, J., Hickman, R. D. G., Marsh, T. R., Rebassa-Mansergas, A., Gänsicke, B. T., Copperwheat, C. M., \& Rodriguez-Gil, P. 2009, A\&A, 507, 929

Southworth, J., Copperwheat, C. M., Gänsicke, B. T., \& Pyrzas, S. 2010a, A\&\&A, 510, A100

Southworth, J., Marsh, T. R., Gänsicke, B. T., Steeghs, D., \& Copperwheat, C. M. 2010b, A\&̋A, $524, \mathrm{~A} 86$ 